\title{
Identification of relaxation functions in glass by mean of a simple experiment
}

\author{
Mathieu Sellier $\uparrow \S$, Norbert Siedow $\ddagger$ and Christopher E. Hann $\dagger$ \\ $\dagger$ Department of Mechanical Engineering, University of Canterbury, Private Bag \\ 4800, Christchurch 8140, New-Zealand \\ $\ddagger$ Fraunhofer-Institut fuer Techno- und Wirtschaftsmathematik (ITWM) \\ Fraunhofer Platz 1, 67661 Kaiserslautern, Germany
}

\begin{abstract}
This communication discusses the possibility of identifying the shear and structure relaxation functions in inorganic glass by means of a single, simple and non-intrusive experiment. The latter consists in measuring the thickness variation over time of a glass plate cooled symmetrically from both sides from a temperature above the glass transition temperature down to room temperature. Results with "artificially" created experimental observations suggest that in spite of its simplicity, the thickness variation curve contains sufficient information to identify uniquely the relaxation functions parameters using the Levenberg-Marquardt method.
\end{abstract}

$\S$ To whom correspondence should be addressed (mathieu.sellier@canterbury.ac.nz)

Based on a paper presented at the $8^{\text {th }}$ ESAFORM conference on material forming in Cluj-Napoca, Romania in 2005.

The authors gratefully acknowledge the funding of the European Union through the Research Training Network MAGICAL (MAthematics for the Glass Industry: Computing and AnaLysis). 


\section{Introduction}

Many numerical techniques are nowadays well established to simulate the thermomechanical behavior of viscoelastic material in complex geometries and under non-trivial mechanical and thermal loadings. However, the potential ability of these techniques to reproduce the reality and therefore provide useful predictive results depends strongly on the quality of the estimate of the material properties.

Glass undergoes stress relaxation upon loading around the glass transition temperature and is also prone to structure relaxation upon cooling, i.e. the state of the structure characterized by the fictive temperature differs depending on the cooling path. Both relaxation phenomena are well described by means of Boltzmann memory integrals, [1], and the challenge is to identify the relaxation moduli and the structure relaxation function (memory kernels). Although the effects of both relaxation phenomena are combined in usual processing conditions, the identification of the memory kernels is often tackled independently. For example, a torsion test is used, on one hand, to identify the shear relaxation modulus at a given temperature (see [2] for a review of the possible methods for measuring the viscoelastic properties of solids). This strategy was used by Lochegnies et al., [3], using experimental data obtained by Kurjian, [4]. The structure relaxation function is found, on the other hand, by imposing a temperature jump to the glass and monitoring the material property variation.

Simultaneous measurement of the stress and structural relaxation functions was performed by DeBast and Gilard, [5], using a differential extensometer. The device allow the simultaneous but independent measurement of the strain resulting from the loading of the sample and the one resulting from stabilization. The experiment considered in the following contrasts by its simplicity. It consists in measuring the thickness variation over time of a glass plate cooled symmetrically from both side from a temperature above 
the glass transition temperature down to room temperature.

The aim of the present work is to investigate whether, in spite of its simplicity, the thickness variation curve contains sufficient information on the stress and structure relaxation phenomena to allow, under several simplifying assumptions, the unique identification of the memory kernels coefficients.

\section{Evaluation of the sample thickness variation}

The thermo-mechanical analysis of a viscoelastic plate in non-isothermal conditions was first performed by Muki and Sternberg, [6]. This solution was later used by Narayanaswamy, [7], to describe tempering of a glass plate. A review of the related work can be found in [8].

\subsection{Constitutive equations}

As a standard starting point of the thermo-mechanical analysis, the glass is pre-supposed to obey, at any location, $\mathbf{x}$, and time, $t$, the following constitutive equations:

$$
\begin{aligned}
& s_{i j}(\mathbf{x}, t)=2 G_{0} \int_{0}^{t} G\left(\xi(\mathbf{x}, t)-\xi\left(\mathbf{x}, t^{\prime}\right)\right) \frac{\partial e_{i j}\left(\mathbf{x}, t^{\prime}\right)}{\partial t^{\prime}} d t^{\prime}, \\
& \sigma(\mathbf{x}, t)=3 K\left(\epsilon(\mathbf{x}, t)-\epsilon_{t h}(\mathbf{x}, t)\right),
\end{aligned}
$$

where $s_{i j}, \sigma$ and $e_{i j}, \epsilon$ are the deviatoric and volumetric parts of the stress and strain tensors, respectively, $\epsilon_{t h}$ the thermal strain, $G_{0}$ the instantaneous shear modulus, and $G$ the shear relaxation modulus. The latter is function of the reduced time $\xi(\mathbf{x}, t)$. Because of the very limited compressibility of inorganic glasses, the bulk modulus $K$ is assumed to be constant, [9]. If, moreover, the influence of the temperature, $T$, on the relaxation behavior can be represented by a classical Arrhenius model, the reduced time 
is expressed as:

$$
\xi(\mathbf{x}, t)=\int_{0}^{t} e^{\frac{\Delta H}{R}\left(\frac{1}{T_{R}}-\frac{\beta}{T\left(\mathbf{x}, t^{\prime}\right)}-\frac{1-\beta}{T_{f}\left(\mathbf{x}, t^{\prime}\right)}\right)} d t^{\prime}
$$

where $\Delta H$ is the activation energy, $R$ the ideal gas constant, $T_{R}$ the reference temperature, and $T_{f}$ the fictive temperature defined as:

$$
T_{f}(\mathbf{x}, t)=T(\mathbf{x}, t)-\int_{0}^{t} M\left(\xi(\mathbf{x}, t)-\xi\left(\mathbf{x}, t^{\prime}\right)\right) \frac{\partial T\left(\mathbf{x}, t^{\prime}\right)}{\partial t^{\prime}} d t^{\prime} .
$$

The shear relaxation modulus, $G$, and structure relaxation function, $M$, are supposed to be well represented by stretched exponential functions, i.e.:

$$
G(\xi)=\exp \left[-\left(\frac{\xi}{\tau_{s}}\right)^{b_{s}}\right] \text { and } M(\xi)=\exp \left[-\left(\frac{\xi}{\tau_{v}}\right)^{b_{v}}\right] .
$$

In eqs. (5), $\tau_{s}>0$ and $\tau_{v}>0$ are the unknown characteristic relaxation times in shear and for the structure, respectively. For most inorganic glasses, the exponent $b_{s}$ is equal or very close to $0.5,[1]$, and this value will be used in the following to reduce the number of unknown parameters. The exponent $b_{v}$, assumed to belong to the interval $[0,1]$, remains to identify. Finally, the thermal strain in eq. (2) is given by:

$$
\epsilon_{t h}(\mathbf{x}, t)=\alpha_{g}\left(T(\mathbf{x}, t)-T_{0}\right)+\left(\alpha_{l}-\alpha_{g}\right)\left(T_{f}(\mathbf{x}, t)-T_{0}\right)
$$

where $\alpha_{g}, \alpha_{l}$ are the coefficients of thermal expansion of the solid and liquid glass, respectively.

\subsection{Reduction to a one-dimensional problem}

If the glass plate has an initial thickness $2 b_{0}$ much smaller than its lateral extent in the $x$ and $y$ directions, the thermo-mechanical problem can be treated as one-dimensional with quantities only depending on the thickness coordinate, $z$, and time.

The sample has initially a uniform temperature $T_{0}$. Heat transfer in semitransparent materials like hot glasses is not only accomplished by conduction but also by radiation, 
which is dominant for high temperatures. The temperature $T(z, t)$ satisfies the radiative heat transfer equation

$$
\begin{aligned}
c_{p} \rho_{g} \frac{\partial T}{\partial t}(z, t)= & \frac{\partial}{\partial z}\left(k_{h} \frac{\partial T}{\partial z}(z, t)\right)-\frac{\partial q}{\partial z}(z, T), \quad T(z, 0)=T_{0}, \\
-k_{h} \frac{\partial T}{\partial z}\left(b_{0}, t\right)= & \alpha\left(T\left(b_{0}, t\right)-T_{a}\right)+ \\
& \varepsilon \pi \int_{\text {opaque }}\left(B_{a}\left(T\left(b_{0}, t\right), \lambda\right)-B_{a}\left(T_{a}, \lambda\right)\right) d \lambda, \\
k_{h} \frac{\partial T}{\partial z}(0, t)= & 0,
\end{aligned}
$$

where $c_{p}$ is the specific heat, $\rho_{g}$ the density of the glass, and $k_{h}$ the thermal conductivity. $\alpha$ denotes the heat transfer coefficient, $T_{a}$ the ambient temperature, and $B_{a}(T, \lambda)$ the Planck function for air depending on temperature and wavelength $\lambda$. $\varepsilon$ is the mean hemispheric surface emissivity.

The radiative flux $q(z)$ is defined as the first moment of the radiative intensity $I(z, \mu, \lambda)$ with respect to the directional cosine $\mu$

$$
q(z)=2 \pi \int_{0}^{\infty} \int_{-1}^{1} I(z, \mu, \lambda) \mu d \mu d \lambda
$$

The intensity satisfies the radiative transfer equation

$$
\begin{aligned}
\mu \frac{\partial I}{\partial z}(z, \mu, \lambda) & =\kappa(\lambda)\left(B_{g}(T(z, t), \lambda)-I(z, \mu, \lambda)\right) \\
I\left(b_{0}, \mu, \lambda\right) & =\rho(\mu) I\left(b_{0},-\mu, \lambda\right)+(1-\rho(\mu)) B_{g}\left(T_{a}, \lambda\right), \mu<0 \\
I(0, \mu, \lambda) & =I(0,-\mu, \lambda), \mu>0 .
\end{aligned}
$$

Here $\kappa(\lambda)$ denotes the absorption coefficient, $\rho(\mu)$ the reflectivity computed using Fresnel law, and $B_{g}(T, \lambda)$ the Planck function for glass.

The one-dimensional assumption and symmetry of the cooling considerably reduce 
the complexity of the mechanical problem. Because the sample is traction free, the in-plane stress must satisfy

$$
\int_{0}^{b_{0}} \sigma_{x x}(z, t)=0
$$

Moreover, for reasons of symmetry well discussed in $[10,11]$, stresses and strains are related as follows:

$$
\begin{aligned}
\sigma_{x x}(z, t)= & 2 \int_{0}^{t} G\left(\xi-\xi^{\prime}\right) \frac{\partial}{\partial t^{\prime}}\left[\epsilon_{x x}\left(t^{\prime}\right)-\epsilon_{z z}\left(z, t^{\prime}\right)\right] d t^{\prime} \\
\sigma_{z z}=0= & \int_{0}^{t}-\frac{4}{3} G\left(\xi-\xi^{\prime}\right) \frac{\partial}{\partial t^{\prime}}\left[\epsilon_{x x}\left(t^{\prime}\right)-\epsilon_{z z}\left(z, t^{\prime}\right)\right]+ \\
& K \frac{\partial}{\partial t^{\prime}}\left[2 \epsilon_{x x}\left(t^{\prime}\right)+\epsilon_{z z}\left(z, t^{\prime}\right)-3 \epsilon_{t h}\left(z, t^{\prime}\right)\right] d t^{\prime}
\end{aligned}
$$

Finally, the half-thickness of the sample, $b(t)$, is obtained using

$$
b(t)=b_{0}+\int_{0}^{b_{0}} \epsilon_{z z}(z, t) d z
$$

\subsection{Solution technique}

For conciseness reasons, only a brief overview of the numerical technique adopted is reported here.

The radiative heat equations (6)-(7) is first solved using a standard implicit Finite Volume technique. For the solution of the radiative transfer equation (eq. (9)), we refer to [15]. The main idea is to split the intensity into two parts $I(z, \mu, \lambda)=$ $I_{1}(z, \mu, \lambda)+I_{2}(z, \mu, \lambda)$, where $I_{1}(z, \mu, \lambda)$ is the solution of the homogeneous equation with inhomogeneous boundary conditions, which can be calculated analytically, and $I_{2}(z, \mu, \lambda)$ is the solution of the inhomogeneous equation with homogeneous boundary conditions, which can be calculated numerically by ray tracing using the formal solution

$$
I_{2}(z, \mu, \lambda)=\kappa(\lambda) \int_{0}^{d(z, \mu)} B_{g}(T(z-s \mu), \lambda) e^{-\kappa(\lambda) s} d s
$$

$d(z, \mu)$ is the distance of the point $z$ to the boundary in direction $-\mu$. 
To simplify the integration of the memory integral (eqs. (4), (13) and (14)), the kernels are first expressed in the form of Prony series using the method proposed by Scherer, [12]. The benefit of this preliminary stage is that, using the algorithm proposed by Taylor et al., [14], the computation of the memory integral no longer requires the entire strain or temperature history but only the values at the previous time step.

The fictive temperature is then obtained by integrating eq. (4) using the stable scheme proposed in [13]. Equation (3) is then integrated with the Trapezoidal Rule to obtain the reduce time. Equations (13) and (14) are discretized in a way similar to that of Taylor et al., [14]. The normal strain variation for the current time step, $\Delta \epsilon_{z z}$, is eliminated from the discrete analog of eq. (13) using eq. (14) so that $\Delta \epsilon_{x x}$ can be evaluated subject to eq. (12).

Results without radiation were validated by comparing the residual stresses with those of the fully three-dimensional simulation performed with the commercial Finite Element code ANSYS. Differences of no more than $5 \%$ were achieved in a fraction of the computational time required by ANSYS.

\subsection{Sample numerical results}

Figure 1 illustrates the variations of the plate thickness, the true, and fictive temperatures for values of the material properties and boundary conditions taken from [8], when available. The initial plate thickness, $b_{0}$, and temperature, $T_{0}$, are $0.305 \mathrm{~cm}$ and $973.15 \mathrm{~K}$. The thermal properties are $\rho_{g}=2537 \mathrm{~kg} / \mathrm{m}^{3}, k_{h}=1.046 \mathrm{~W} / \mathrm{mK}, c_{p}=3349.44$ $\mathrm{J} / \mathrm{kgK}$, and the adsorption coefficient, $\kappa(\lambda)$, is set to $0.3 \mathrm{~cm}^{-1}$ for $1<\lambda<2.7 \mu \mathrm{m}$, $5 \mathrm{~cm}^{-1}$ for $2.7<\lambda<4.5 \mu \mathrm{m}$, and $\infty$ for $\lambda>4.5 \mu \mathrm{m}$ while a value of 1.46 is chosen for the refraction index. The kernels are defined by the parameters $\left(\tau_{s}, b_{s}\right)=(577,0.5)$, $\left(\tau_{v}, b_{v}\right)=(6350,0.68)$ at the reference temperature $T_{R}=791.45 \mathrm{~K}$ and the mechanical properties correspond to $K=40.5 \mathrm{GPa}, G_{0}=28 \mathrm{GPa}, \alpha_{l}=33.6 \times 10^{-6} \mathrm{~K}^{-1}, \alpha_{g}=11.2 \times 10^{-6}$ 


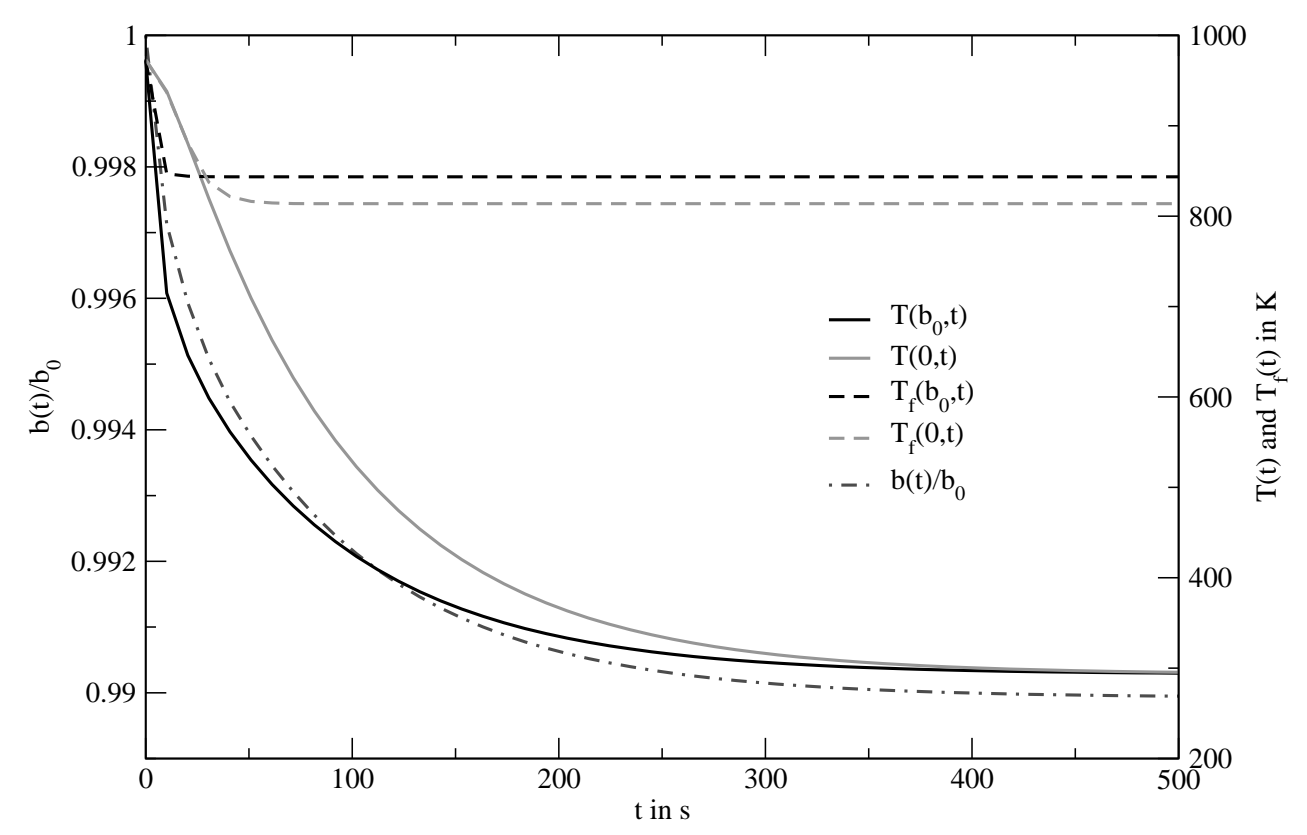

Figure 1. Time variation of the plate thickness, the true, and fictive temperatures at the surface and centerline of the plate.

$\mathrm{K}^{-1}$. The required parameters to define the reduced time in eq. (3) are set to $\Delta H=150$ $\mathrm{kcal} / \mathrm{mol}, R=1.987 \mathrm{cal} / \mathrm{molK}$, and $\beta=0.5$. Finally, the heat transfer coefficient $\alpha=468.6$ $\mathrm{W} / \mathrm{m}^{2} \mathrm{~K}$ with an ambient air temperature $T_{a}=293.15 \mathrm{~K}$ are representative of a rapid tempering of the plate. This rapid tempering induces large temperature gradients in the plate resulting the strong contrast of the fictive temperature between the surface and the core of the plate shown in Figure 1. Moreover, the steepening of the temperature decrease, apparent in Figure 1, confirms the importance of the radiative heat transfer at high temperature.

\section{Kernel parameter identification}

The definition of the identification problem is the following: "identify the shear and structure relaxation parameters $\left(\tau_{s}, \tau_{v}, b_{v}\right)$ in eqs. (5) so that the calculated response (the sample thickness variation, $b(t)$ ) matches in a least square sense the experimental 
observations, $b^{o b s}(t)$ ", i.e.:

$$
\min _{p \in p_{\text {adm }}} E(p)=\frac{1}{2} \sum_{i=1}^{l} r_{i}^{2}(p)=\frac{1}{2} \sum_{i=1}^{l}\left(b\left(t_{i}\right)-b^{o b s}\left(t_{i}\right)\right)^{2},
$$

where $E$ is the error function, $l$ is the number of observation points and the vector parameter $p=\left(\tau_{s}, \tau_{v}, b_{v}\right)$ must belong to the space of admissible parameters, $p_{a d m}$. The latter include all the vectors with the values of $\tau_{s}, \tau_{v}, b_{v}$ which satisfy the constraints given in section 2.1 .

Husain and Anderssen recently proposed algorithms to recover the parameters of the relaxation moduli, expressed in the form of stretched exponential (or Kohlrausch) functions, using moments of the measured stress and the corresponding known applied strain rate, $[16,17]$, but the idea is not straight-forward to extend to the non-isothermal problem. Instead, the popular Levenberg-Marquardt method, [18], which combines the benefits of the Newton and steepest descent methods is used to minimize $E$ in eq. (16). It determines iteratively the necessary correction $d p^{k}$ to the vector parameter $p$ according to

$$
\left[\left(J^{(k)}\right)^{T} J^{(k)}+\beta^{(k)} I\right] d p^{k}=-\left(J^{(k)}\right)^{T} r^{(k)} .
$$

$J^{(k)}$ is the Jacobian matrix of $E$ calculated by Finite Differences and $\beta^{(k)}$ is a positive constant chosen equal to $10^{-3}$ initially. If the current iteration successfully decreases $E, \beta^{(k)}$ is divided by 10 , it is multiplied by 10 otherwise. The linear system of equations (17) is solved in MATLAB for $d p^{(k)}$ and the vector parameter is updated according to $p^{(k+1)}=p^{(k)}+d p^{(k)}$. In order to transform the constrained least-square problem into an unconstrained one, a variable transformation is performed. The choice of transformed variables $\tilde{p}=\left(p_{1}, p_{2}, p_{3}\right)$ with $\left(p_{1}^{2}, p_{2}^{2}, \sin ^{2}\left(p_{3}\right)\right)=\left(\tau_{s}, \tau_{v}, b_{v}\right)$ naturally enforces the constraints on $\tau_{s}, \tau_{v}$ and $b_{v}$. 


\section{Results and analysis}

Unfortunately, true experimental results are not available. Nevertheless, in order to assess the method, tests are performed with an "artificial" experimental thickness variation, $b^{\text {obs }}\left(t_{i}\right)$, obtained numerically for the values of the parameters reported previously, i.e. $\quad p^{o b s}=\left(\tau_{s}^{o b s}, \tau_{v}^{o b s}, b_{v}^{o b s}\right)=(577,6350,0.67)$ at $T_{R}=791.45 \mathrm{~K}$. Noise is introduced by adding a random component of magnitude 2 mag to the smooth experimental curve according to

$$
b^{o b s}(t)=(2 \operatorname{mag} * \operatorname{rand}(t)+(1-\operatorname{mag})) b(t), \operatorname{rand}(t) \in[0,1] .
$$

A noise magnitude of $1.2 \times 10^{-4} b_{0}$ is chosen. This corresponds to an uncertainty of $1 \%$ on the experimental readings since the overall thickness variation, shown in Figure 1 , is around $1.2 \times 10^{-2} b_{0}$. Note that for a $1 \mathrm{~cm}$ thick glass plate, this uncertainty is on the order of one micron which is well within the precision range of laser sensors $\|$. The Levenberg-Marquardt algorithm is stopped after $k_{\text {stop }}$ iterations when a maximum of 200 iterations is reached or $\beta^{(k)}$ in eq. (17) becomes greater than $10^{25}$ as experience showed no further changes in the solution beyond these thresholds.

For three different coefficients of heat transfer $\left(\alpha=468.6,280.32\right.$ and $\left.92 \mathrm{~W} / \mathrm{m}^{2} \mathrm{~K}\right)$, the Levenberg-Marquardt algorithm is launched 30 times from different starting points in the parameter space, $p^{0}=\left(\tau_{s}^{0}, \tau_{v}^{0}, b_{v}^{0}\right)$, and with different noise patterns on the observed sample thickness variation. Table 1 reports the mean and standard deviation of the resulting, identified parameters based on these 30 runs. Although not precisely identical, the average values of the identified parameters are reasonably close to the observed ones, $p^{o b s}=(577,6350,0.67)$, for all the coefficients of heat transfer. Note that for a noiseless thickness variation, the Levenberg-Marquardt algorithm only fails for unrealistic initial || See, for example, the Keyence sensor range at http://world.keyence.com 




Figure 2. Residual stress profiles: Experimental (line with dot symbols); Reconstructed using using 10 different sets of identified parameters (solid lines) for $\alpha=468.6 \mathrm{~W} / \mathrm{m}^{2} \mathrm{~K}$.

guesses. The standard deviation in Table 1 is quite large but Figure 2 and Table 1 reveal that this scatter of the identified parameters has little influence on the residual stresses at room temperature. Indeed, the maximum, relative, residual stress difference between the experimental and reconstructed curves defined as $\max _{z \in\left[0, b_{0}\right]}\left\|\frac{\sigma_{x x}\left(z, t_{f}\right)-\sigma_{x x}{ }^{o b s}\left(z, t_{f}\right)}{\sigma_{x x}{ }^{o b s}\left(0, t_{f}\right)-\sigma_{x x}{ }^{o b s}\left(b_{0}, t_{f}\right)}\right\|$, where $t_{f}$ is the final time when the temperature is uniformly equal to room temperature, never exceeds $10 \%$ and the experimental and reconstructed residual stress profiles plotted in Figure 2 are almost undistinguishable.

Finally, although the choice to set the value of $\beta$ in eq. (3) to 0.5 is the most natural one and the one made by Narayanaswamy in its seminal paper, [7], this choice is not unique and somehow arbitrary. A possible strategy to remove this indetermination is to run the Levenberg-Marquardt algorithm for several values of $\beta$ and retain the one 
which minimizes the error function $E$. To test this strategy, "artificial" experimental data are created with $\beta=\beta^{o b s}=0.5, \alpha=92 \mathrm{~W} / \mathrm{m}^{2} \mathrm{~K}$ and without any noise. The Levenberg-Marquardt is then run with $\beta$ set to $0.2,0.3,0.4,0.5,0.6,0.7$, and 0.8 and the corresponding, computed values of $E$ are found to be $2.7 \times 10^{-10}, 1.8 \times 10^{-11}$, $2 \times 10^{-12}, 1.6 \times 10^{-22}, 6.6 \times 10^{-13}, 9.29 \times 10^{-10}$ and $7.5 \times 10^{-9}$, respectively. Clearly, $\beta=0.5$ minimizes the error function and is therefore, as expected, the sought value.

\section{Conclusions}

The present results demonstrate, at least in theory, the possibility of identifying the shear relaxation modulus and the structure relaxation function through the measurement of the time variation of the glass plate thickness. The identified parameters are close to the experimental ones on average but present quite a large scatter. However, the effect on the residual stresses is small and might be reduced by measuring the thickness variation of two plates with different thicknesses and fitting the results simultaneously. Only comparison with real experimental data can guaranty the feasibility of the method but a number of potential benefits may be outlined. Although the independent identification of the shear relaxation modulus and the structure relaxation function is probably more accurate, both effects are normally combined in practice and the suggested experiment is therefore more representative of real processing conditions. Moreover, the method is non-intrusive and the thickness variation can be measured in practice with a high degree of accuracy and with little noise.

\section{Acknowledgment}

The authors wish to thank G.W. Scherer for his insightful remarks and suggestions on an earlier draft of the manuscript. 


\section{References}

[1] G.W. Scherer, "Relaxation in glass and composites"; Edited by Wiley, New York, 1986.

[2] R.S. Lakes, "Viscoelastic measurement techniques," Rev.Sci. Instrum., 75 797-810 (2004).

[3] D. Lochegnies, P. Wiertel, and O.S. Narayanaswamy, "Inverse determination of material properties and optimization of flat glass tempering," Int. J. Form. Proc., 2 95-116 (1999).

[4] C.R. Kurjian, "Relaxation of torsional stress in the transformation range of a soda-lime-silica glass," Phys. Chem. Glasses, 4 128-136 (1963).

[5] J. DeBast and P. Gilard, "Variation of the viscosity of glass and the relaxation of stresses during stabilization," Phys. Chem. Glasses, 4 117-128 (1963).

[6] R. Muki and E. Sternberg, "On transient thermal stresses in viscoelastic materials with temperature-dependent properties," J. Appl. Mech., June 193-207 (1961).

[7] O.S. Narayanaswamy, "Stress and structural relaxation in tempering glass," J. Am. Ceram. Soc., 61 146-152 (1978).

[8] O.S. Narayanaswamy, "Annealing of glass"; chap. 5 in Glass science and technology, vol. 3, Edited by D.R. Uhlmann and N.J. Kreidl. Academic Press (1986).

[9] R. Gardon, "Thermal tempering of glass"; chap. 5 in Glass science and technology, vol. 5, Edited by D.R. Uhlmann and N.J. Kreidl. Academic Press (1980).

[10] W. Potze, F.H. in't Veld, and J.P. van den Brink, "Structural and stress relaxation in a glass slab," Int. J. Form. Proc., 2 63-80 (1999).

[11] X. Guo and A.I. Isayev, "Thermal residual stresses in freely quenched slabs of semicrystalline polymers: simulation and experiment," J. Appl. Polym. Sci., 75 1404-1415 (2000).

[12] G.W. Scherer, "Volume relaxation far from equilibrium," J. Am. Ceram. Soc., 69 374-381 (1986).

[13] A. Markovsky and T. Soules, "An efficient and stable algorithm for calculating fictive temperatures," C. Am. Ceram. Soc., 67 56-57 (1984).

[14] R.L. Taylor, K.S. Pister, and G.L. Goudreau, "Thermomechanical analysis of viscoelastic solids," Int. J. Numer. Meth. Eng., 2 45-59 (1970).

[15] N. Siedow, T. Grosan, D. Lochegnies, and E. Romero, "Application of a new method for radiative heat transfer to flat glass tempering," J. Am. Ceram. Soc., 88 2181-2187 (2005).

[16] S.A. Husain and R.S. Anderssen, "Modelling the relaxation modulus of linear viscoelasticity using Kohlrausch functions," J. Non-Newtonian Fluid Mech., 125 159-170 (2005).

[17] S.A. Husain and R.S. Anderssen, "Algorithms for the recovery of Kohlrausch parameters from 

viscoelastic stress-strain data," ANZIAM J., 46 C935-C955 (2005).

[18] J. Nocedal and S.J. Wright, "Numerical optimization"; Edited by Springer, New-York, 1999. 


\section{Figure caption}

Figure 1: Time variation of the plate thickness, the true, and fictive temperatures at the surface and centerline of the plate.

Figure 2: Residual stress profiles: Experimental (line with dot symbols); Reconstructed using 10 different sets of identified parameters (solid lines) for $\alpha=468.6 \mathrm{~W} / \mathrm{m}^{2} \mathrm{~K}$. 


\section{Table}

Table 1. Parameters identification statistics $\Phi$.

\begin{tabular}{|c||c|c|c|}
\hline$\alpha$ in $\mathrm{W} / \mathrm{m}^{2} \mathrm{~K}$ & $\operatorname{mean}\left(p^{k_{\text {stop }}}\right)$ & $\operatorname{std}\left(p^{k_{\text {stop }}}\right)$ & $\begin{array}{c}\text { Maximum , relative, residual } \\
\text { stress difference in } \%\end{array}$ \\
\hline 468.6 & $(638,6615,0.7)$ & $(377,0.13,737)$ & 5.9 \\
\hline 280.32 & $(784,6086,0.68)$ & $(534,0.1,1756)$ & 10.8 \\
\hline 92 & $(774,6225,0.74)$ & $(739,0.18,815)$ & 10 \\
\hline
\end{tabular}

ฯ Mean and standard deviation of the resulting, identified parameters based on 30 runs. The maximum, relative, residual stress difference is defined as $\max _{z \in\left[0, b_{0}\right]}\left\|\frac{\sigma_{x x}\left(z, t_{f}\right)-\sigma_{x x}{ }^{o b s}\left(z, t_{f}\right)}{\sigma_{x x}{ }^{o b s}\left(0, t_{f}\right)-\sigma_{x x}{ }^{o b s}\left(b_{0}, t_{f}\right)}\right\|$, where $t_{f}$ is the final time when the temperature is uniformly equal to room temperature. 